\title{
The Eventual Intracellular Destruction of Staphylococci by Mononuclear Cells
}

\author{
By M. G. SHAYEGANI* AND F. A. KAPRAL \\ The Department of Microbiology, School of Medicine, \\ University of Pennsylvania, Philadelphia, U.S.A.
}

(Received 20 February 1962)

\section{SUMMARY}

It was found that Staphylococcus aureus usually survived within monocytes of normal rabbits for several hours without multiplication, but were eventually destroyed. However, there was variation in the intracellular behaviour in leucocytes of different rabbits in that cells from some donors began the slow destruction of the staphylococci shortly after phagocytosis.

In many of these experiments streptomycin was incorporated in the tissue-culture medium to suppress the extracellular multiplication of staphylococci. Interference by streptomycin with the intracellular behaviour of staphylococci was considered as minimal on the basis that the intracellular survival was not influenced by different concentrations of the drug in the tissue-culture medium nor did incubation of the cells in the antibiotic before infection alter the subsequent survival of the organisms.

\section{INTRODUCTION}

In a previous study (Kapral \& Shayegani, 1959) it was reported that Staphylococcus aureus could survive for several hours within rabbit mononuclear leucocytes. No multiplication or destruction of staphylococci was observed during this interval with the methods used. Subsequently attempts were made to determine the eventual fate of the surviving intracellular staphylococci.

\section{METHODS}

Staphylococcal strain. Staphylococcus aureus strain 18-Z; the characters of this strain were described by Kapral \& Shayegani (1959). A mutant strain 18-Z, SMr resistant to $1000 \mu \mathrm{g}$. streptomycin $/ \mathrm{ml}$. medium was isolated from the parent $18-\mathrm{Z}$ strain. The mutant appeared identical with the parent strain in all other in vitro characteristics.

Monocytes. Rabbit mononuclear exudate cells were prepared as described by Kapral \& Shayegani (1959).

\footnotetext{
* Present address : Department of Clinical Pathology, Philadelphia General Hospital, Philadelphia, Pennsylvania, U.S.A.
} 


\section{Tissue culture procedures}

Plastic chamber method. This tissue-culture procedure was the same as previously described (Kapral \& Shayegani, 1959) with the exception that $0.2 \mathrm{ml}$. of $10 \%$ sodium bicarbonate solution was incorporated into the tissue-culture medium and the chambers placed under $5 \% \mathrm{CO}_{2}$ to stabilize the $\mathrm{pH}$ at $7 \cdot 4$ during the long incubation period. Fifty $\mu \mathrm{g}$. streptomycin $/ \mathrm{ml}$. medium was used in these experiments.

Suspended cultures. Suspensions of mononuclear leucocytes and Staphylococcus aureus 18-Z were prepared as previously described (Kapral \& Shayegani, 1959). About $2 \times 10^{8}$ monocytes and $5 \times 10^{9}$ staphylococci were suspended in $1 \mathrm{ml}$. of $10 \%$ normal rabbit serum in Hanks solution. The mixture was centrifuged at $100 \mathrm{~g}$ for 3 min., then placed in a water bath at $37^{\circ}$ for 5 min. The sedimented infected cells were washed three times with $10 \mathrm{ml}$. quantities of cold Hanks solution containing $10 \%$ normal rabbit serum. Samples of the washed infected cells were then placed in siliconized $50 \mathrm{ml}$. flasks containing $10 \mathrm{ml}$. of $10 \%$ normal rabbit serum in Hanks solution at $\mathrm{pH} \mathbf{7 \cdot 2}$. The suspensions were kept agitated by Teflon stirring bars driven by a magnetic stirrer. After $1 \mathrm{hr}$. incubation streptomycin was added to medium to give a final concentration of $50 \mu \mathrm{g} . / \mathrm{ml}$. Samples were removed and treated according to Cohn \& Morse (1959) with the exception that the cells were lysed by $5 \%$ saponin solution and that counts of viable (trypan blue negative) leucocytes were also done on each sample.

\section{RESULTS}

A series of experiments was performed with normal rabbit monocytes infected with Staphylococcus aureus $18-\mathrm{Z}$ in plastic tissue-culture chambers.

Figure 1 illustrates some representative results. There was considerable variation in the intracellular survival of $S$. aureus $18-Z$ within monocytes of different rabbits. In one case (A) there was a persistent survival for $30 \mathrm{hr}$. In another instance (B) there was a period of survival followed by destruction. In still other rabbit monocytes (C, D) staphylococci were destroyed without any evidence of a period of survival.

In many experiments the serum of the rabbit donating the cells as well as the rabbit serum used in the tissue-culture medium was tested for the presence of antibody against soluble products of the test organism by the method of Elek \& Levy (1950). In these cases no detectable precipitating antibodies were found; therefore, the rabbits were assumed to be normal.

Different multiplicity of infection. Since it was possible that the late destruction of strain 18-Z might somehow be dependent on the number of organisms per cell, experiments were performed with different multiplicities of infection using cells from the same rabbit. It was noted that the eventual destruction of intracellular staphylococci did not depend on the multiplicity of infection. This is illustrated in Fig. 2 where multiplicities of 25 and 100 staphylococci per monocyte were used to yield different initial infectivities, yet the fate of the organisms was the same in both instances.

Destruction within monocytes maintained in suspension. Studies using suspended 


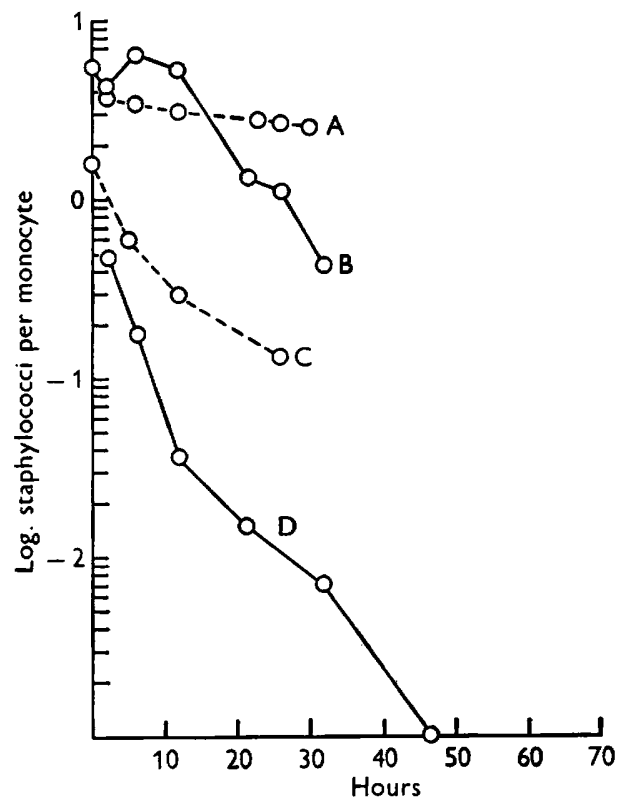

Fig. 1. Long-term survival of Staphylococcus aureus strain 18-Z within monocytes derived from different rabbits (A-D).

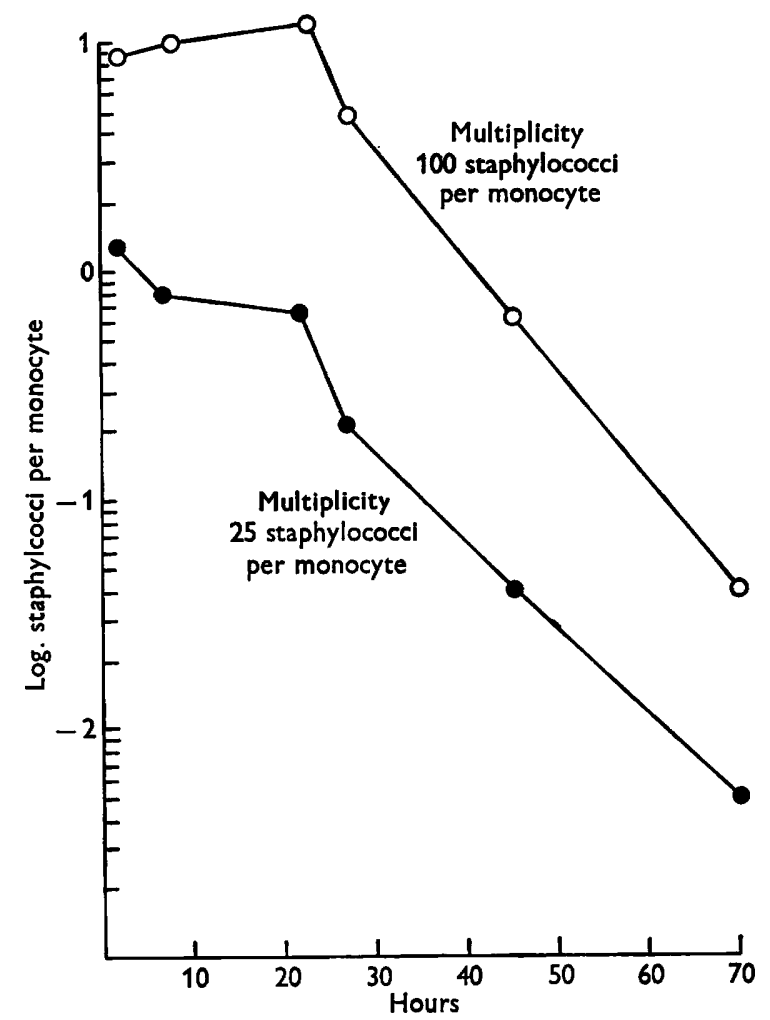

Fig. 2. Intracellular survival of Staphylococcus aureus strain 18-Z with different multiplicities of infection. 
cultures gave similar results as obtained in the plastic chambers. Fig. 3 illustrates the results obtained in an experiment where infected monocytes were maintained for $50 \mathrm{hr}$. After a short period of survival the staphylococci were slowly destroyed.

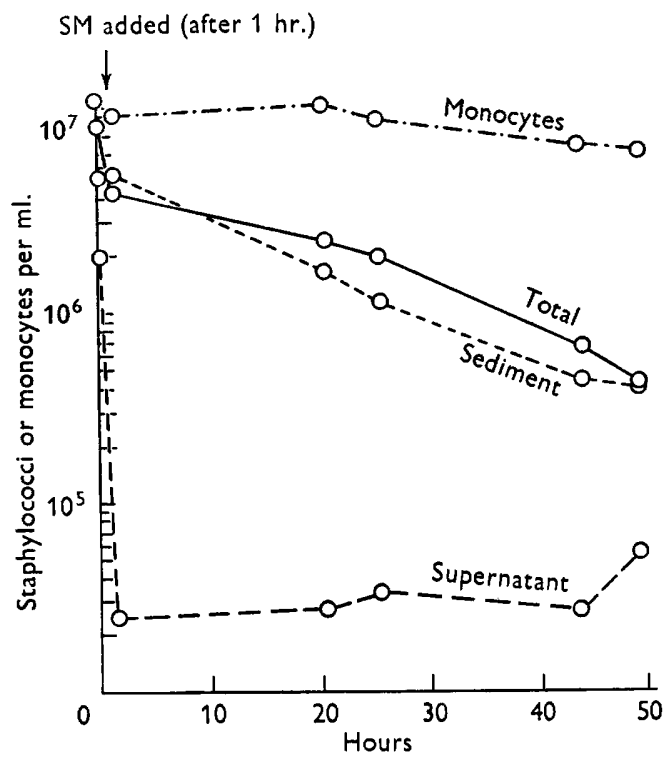

Fig. 3. Survival of Staphylococcus aureus strain 18-Z within rabbit monocytes maintained in suspension.

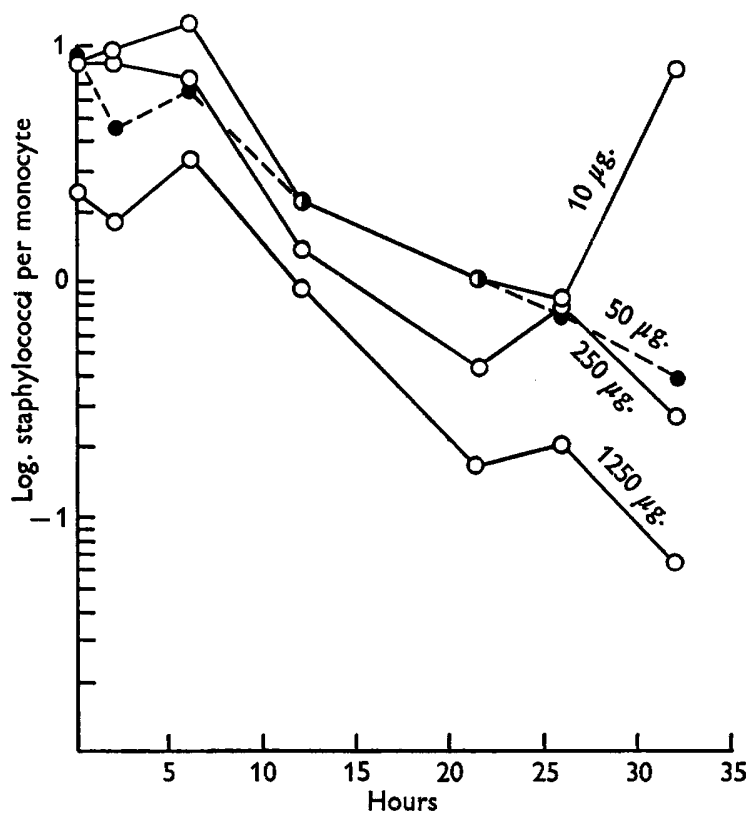

Fig. 4. The survival of Staphylococcus aureus strain 18-Z within monocytes in the presence of various concentrations of streptomycin. 
Since streptomycin was routinely incorporated into the tissue-culture medium, there was a possibility that this antibiotic might penetrate into the monocytes, which could in turn account for the intracellular destruction of staphylococci.

The following experiments are reported since the data tend to indicate that streptomycin does not seriously interfere with the intracellular behaviour of staphylococci.

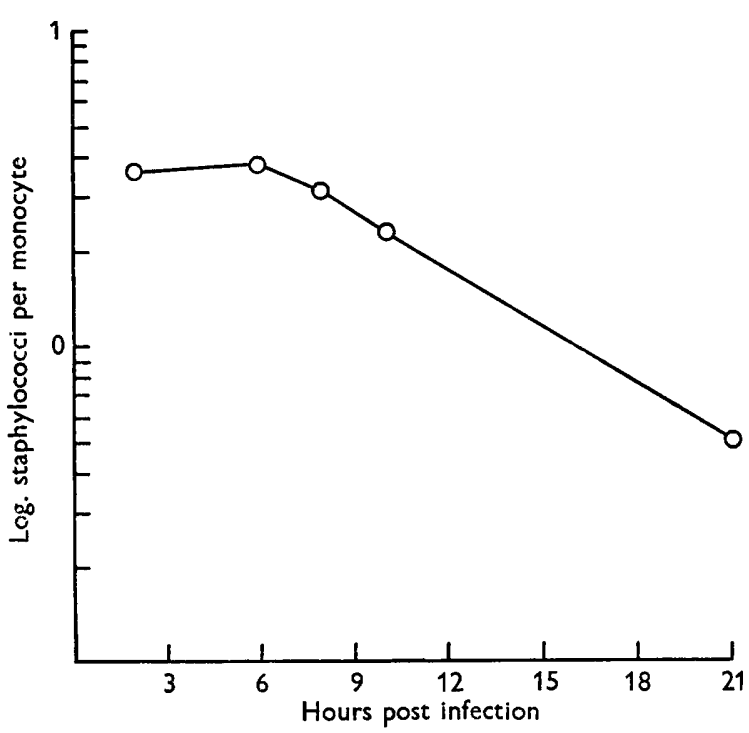

Fig. 5

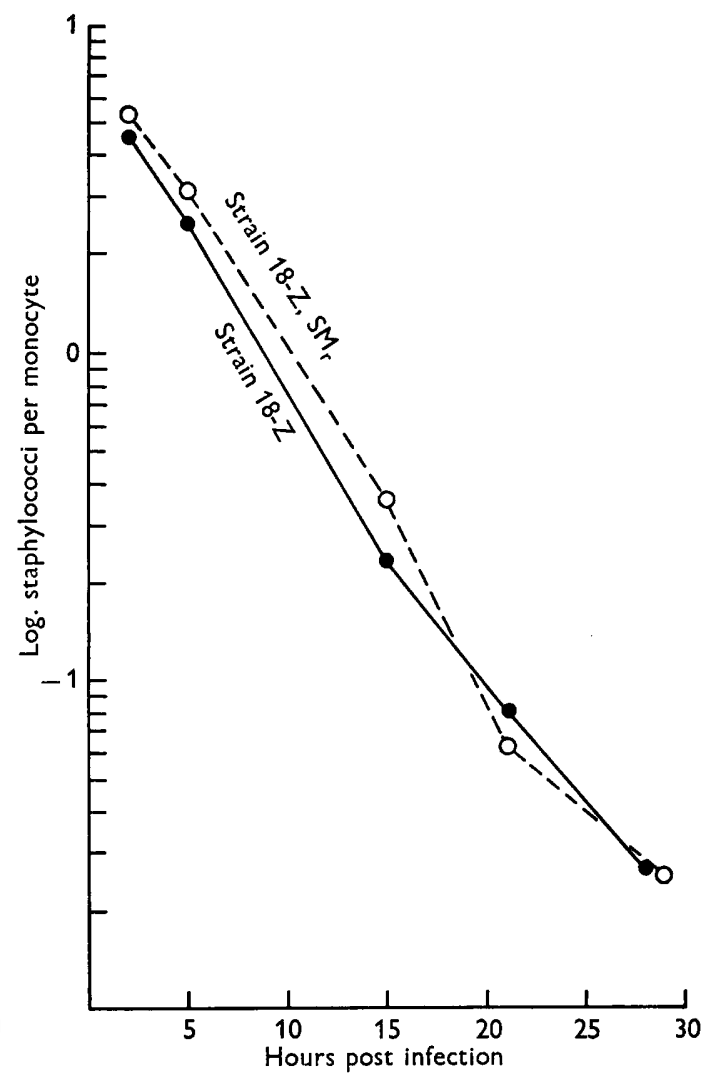

Fig. 6

Fig. 5. Survival of Staphylococcus aureus strain $18-\mathrm{Z}$ within monocytes maintained in the presence of streptomycin before infection.

Fig. 6. Survival of Staphylococcus aureus within monocytes maintained in the presence of streptomycin before infection with strain $18-\mathrm{Z}, \mathrm{SM}_{\mathbf{r}}$.

Different concentrations of streptomycin in tissue-culture medium. A series of experiments was performed with monocytes from one rabbit to compare the effect of different concentrations of streptomycin in the tissue-culture medium on the rate of destruction of intracellular staphylococci. It seemed reasonable to assume that if the streptomycin would slowly penetrate into the monocytes, a higher concentration in the tissue-culture medium would increase the rate of penetration and perhaps increase the rate of killing of intracellular staphylococci. Fig. 4 illustrates the results obtained under the conditions in which $10,50,250$ and $1250 \mu \mathrm{g}$. streptomycin/ml. medium were used. Under these conditions the intracellular destruction 
in the presence of $\mathbf{1 0 - 2 5 0} \mu \mathrm{g} . / \mathrm{ml}$. was essentially the same. It should be noted, however, that after $26 \mathrm{hr}$., streptomycin in a concentration of $10 \mu \mathrm{g} . / \mathrm{ml}$. medium no longer suppressed the extracellular multiplication of $S$. aureus 18-Z. It was because of such failures of low concentrations of streptomycin reliably to control the extracellular population of the strain that higher concentrations were routinely used. When $1250 \mu \mathrm{g}$. streptomycin/ml. medium was used, there appeared to be a slightly greater decline in the intracellular staphylococci, but it is difficult to say whether this is significant.

Delayed infection of monocytes. It was also thought that if the intracellular staphylococci were destroyed by the slow penetration of streptomycin rather than by the innate capacity of the monocytes, it might result in increased killing if the monocytes were allowed to equilibrate with streptomycin before infection.

It was found that monocytes adhering to coverslips in tissue-culture chambers for some 24-30 hr. (in medium containing $50 \mu \mathrm{g}$. streptomycin $/ \mathrm{ml}$. medium) could readily phagocytize organisms which were introduced into the chamber.

Fig. 5 shows that the behaviour of staphylococci within monocytes kept in medium containing streptomycin for $24 \mathrm{hr}$. before infection was similar to that found if phagocytosis had occurred immediately.

Infection with streptomycin-resistant mutant. Monocytes from one rabbit were placed in tissue culture in the presence of $50 \mu \mathrm{g}$. streptomycin $/ \mathrm{ml}$. for $30 \mathrm{hr}$. At this time the cells were infected with either strain $18-\mathrm{Z}$ or the streptomycin-resistant strain 18-Z SM. Streptomycin $(50 \mu \mathrm{g} . / \mathrm{ml}$.) was present in the medium in both cases, but in cultures infected with the streptomycin-resistant mutant, $40 \mu \mathrm{g}$. Kanamycin $/ \mathrm{ml}$. was also added to control extracellular multiplication. It will be seen (Fig. 6) that these strains behaved similarly within the cells; therefore, it is not likely that the killing was due to the presence of streptomycin within the leucocytes. However, since Kanamycin was used to control the extracellular streptomycin-resistant mutants a possibility remains that the leucocytes became 'permeable' to both drugs after a time and the destruction actually resulted from the antibiotics. It may also be noticed that the organisms were killed early and continually in these particular cells.

\section{DISCUSSION}

Tissue-culture methods offer an experimental approach to the study of intracellular bacterial pathogens. However, the conditions are such that extracellular multiplication of the organisms can be misinterpreted as intracellular multiplication if continuous phagocytosis by the host cells proceeds. Antibiotics (e.g. streptomycin) were incorporated into the tissue-culture medium in order to reduce this possibility by inhibiting extracellular multiplication. However, the possibility remains that the antibiotic so used may penetrate the host cells and thereby become bactericidal or bacteriostatic for intracellular organisms. There is no obvious direct experimental method to disprove such a possibility. It appears very likely that streptomycin enters leucocytes by means of pinocytosis, but this does not necessarily imply that the antibiotic can actually come into contact with the intracellular organism. The membrane about the vacuoles might still prevent penetration of the 
antibiotic into cytoplasm or the vacuole containing the organism. However, indirect experiments were performed to determine any gross effects of streptomycin on the behaviour of the intracellular staphylococci. The removal of most of the extracellular organisms by repeated washing gave results similar to those when streptomycin was used, but this procedure was impractical for long periods. Furthermore, the intracellular behaviour was not critically dependent on the streptomycin concentration in the medium, nor did pre-incubation of cells in the antibiotic alter the fate of the phagocytized staphylococci. The fact that tubercle bacilli and brucella can multiply within monocytes in the presence of streptomycin (Suter, 1953; Hsu \& Kapral, 1960; Berthrong \& Hamilton, 1959; Pomales-Lebron \& Stinebring, 1957; Murate, Stinebring, Schaffner \& Lechevalier, 1959) lends support to the idea that this antibiotic does not drastically affect intracellular organisms.

The results reported here also indicate that the intracellular behaviour of staphylococci may differ in leucocytes from different rabbits. Since no detectable precipitating antibody was demonstrated in the sera of these animals it was presumed that these rabbits had had no recent major contact with the organism. This variation suggests the possibility that there may be an inherent difference in the leucocytes from different donors, but the variation might also result from subtle differences in the manipulation of the cell preparations.

The prolonged intracellular survival of Staphylococcus aureus might explain part of the pathogenesis of staphylococcal infections. Kapral \& $\mathrm{Li}(1960)$ and $\mathrm{Li} \&$ Kapral (1962) showed that $S$. aureus $18-Z$ (and derived coagulase negative mutants) when injected intravenously into rabbits survived (without apparent multiplication) in the lungs, liver, and spleen for as long as $\mathbf{2 - 3}$ weeks. It is possible that the organisms persist within reticulo-endothelial cells of these organs, but proof of this is lacking.

This research was supported by funds received from United States Public Health Service Grant E-2695.

\section{REFERENCES}

Berthrong, M. \& Hamiton, M. A. (1959). Tissue culture studies on resistance in tuberculosis. II. Monocytes from normal and immunized guinea pigs infected with virulent human tubercle bacilli. Amer. Rev. Tuberc. 79, 221.

CoHN, Z. A. \& Morse, S. I. (1959). Interaction between rabbit polymorphonuclear leucocytes and staphylococci. J. exp. Med. 110, 419.

ELEK, S. D. \& LEvY, D. (1950). Diffusible antigens in staphylococcal cultures. Brit. $J$. exp. Path. 31, 358.

Hsu, H. S. \& KaPraL, F. A. (1960). The suppressed multiplication of tubercle bacilli within macrophages derived from triiodothyronine-treated guinea pigs. Amer. Rev. Tuberc. 91, 881.

Kapral, F. A. \& Shayegani, M. G. (1959). Intracellular survival of staphylococci. $J$. exp. Med. 110, 123.

KaPraL, F. A. \& LI, I. W. (1960). Virulence and coagulases of Staphylococcus aureus. Proc. Soc. exp. Biol., N.Y. 104, 151.

LI, I. W. \& KapraL, F. A. (1962). Virulence and coagulases of Staphylococcus aureus. II. Survival of certain coagulase negative mutants in the organs of intravenously infected rabbits. J. infect. Dis. (in the Press). 
Murate, A. M., Stinebring, W. R., Schaffner, C. P. \& Lechevalier, H. (1959). Screening for antibiotics active against intracellular bacteria. Appl. Microbiol. 7, 109.

Pomales-Lebron, A. \& Stinebring, W. R. (1957). Intracellular multiplication of Brucella abortus in normal and immune phagocytes. Proc. Soc. exp. Biol., N.Y. 94, 78.

SUTER, E. (1953). Multiplication of tubercle bacilli within mononuclear phagocytes in tissue cultures derived from normal animals and animals vaccinated with BCG. $J$. exp. Med. 97, 235. 